\title{
Phase II clinical study of neoadjuvant chemotherapy with CDDP/ CPT-11 regimen in combination with radical hysterectomy for cervical cancer with a bulky mass
}

\author{
Tadahiro Shojii ${ }^{1}$ Eriko Takatori $^{1} \cdot$ Yoko Furutake $^{1} \cdot$ Anna Takada $^{1}$ • \\ Takayuki Nagasawa ${ }^{1} \cdot$ Hideo Omi $^{1} \cdot$ Masahiro Kagabu $^{1} \cdot$ Tatsuya Honda $^{1}$. \\ Fumiharu Miura $^{1}$ - Satoshi Takeuchi ${ }^{1}$ Seisuke Kumagai ${ }^{2}$ Akira Yoshizaki ${ }^{3}$. \\ Akira Sato $^{4} \cdot$ Toru Sugiyama ${ }^{1}$
}

Received: 2 March 2016 / Accepted: 14 June 2016 / Published online: 24 June 2016

(c) The Author(s) 2016. This article is published with open access at Springerlink.com

\begin{abstract}
Background We examined the efficacy and safety of neoadjuvant chemotherapy (NAC) with the CPT-11 + CDDP regimen in combination with radical hysterectomy.

Subjects and methods The subjects were 42 patients with stages IB2 to IIIB squamous cell carcinoma of the uterine cervix with a bulky mass. CDDP at $70 \mathrm{mg} / \mathrm{m}^{2}$ was intravenously administered on day 1 and CPT- 11 at $70 \mathrm{mg} / \mathrm{m}^{2}$ was intravenously administered on days 1 and 8 of a 21-day cycle. In principle, two cycles were administered followed by radical hysterectomy. We examined antitumor efficacy, adverse events, completion rate of radical hysterectomy, operative time, surgical blood loss, progression-free survival (PFS), and overall survival (OS).

Results The antitumor effect was complete response in 7 patients, partial response in 28 , stable disease in 6 , and progressive disease in 1; the response rate was $83.3 \%$ (95\% confidence interval, 68.6-93.0). Grade 3 or more severe neutropenia, anemia, and platelet count decreases were noted in $23(54.8 \%), 4(9.5 \%)$, and $1(2.4 \%)$ patient, respectively. Grade 3 nausea occurred in 3 patients $(7.1 \%)$, vomiting in $1(2.4 \%)$, and grade 3 febrile neutropenia in 2 $(7.1 \%)$. The completion rate of radical hysterectomy was
\end{abstract}

Tadahiro Shoji

tshoji@iwate-med.ac.jp

1 Department of Obstetrics and Gynecology, Iwate Medical University School of Medicine, 19-1 Uchimaru, Morioka 020-8505, Japan

2 Department of Obstetrics and Gynecology, National Hospital Organization Kokura Medical Center, Kokura, Japan

3 Department of Gynecology, Miyama Hospital, Oshu, Japan

4 Department of Gynecology, Medical Coat Hachinohe West Hospital, Hachinohe, Japan
$88.1 \%$. The median operative time and surgical blood loss were $260 \mathrm{~min}$ (range, 210-334) and $500 \mathrm{ml}$ (range, 393898 ), respectively. The 5-year PFS rate was $67.2 \%$, and the 5-year OS rate was $68.0 \%$. In multivariate analysis, lymph node metastasis before NAC [hazard ratio (HR), 34.88] and non-response to NAC (HR 30.58) were significant prognostic factors.

Conclusion NAC with the CDDP/CPT-11 regimen achieves a high antitumor efficacy with moderate adverse reactions, allowing safe radical hysterectomy, and is thus considered to be a useful therapeutic method that can improve prognosis.

Keywords Cervical cancer · Bulky mass - NAC · CDDP . CPT-11

\section{Introduction}

Therapeutic methods for stage IB2 to IIB cervical cancer with a bulky mass differ between Japan and Western countries. Based on the results of large-scale randomized studies and meta-analyses, concurrent chemoradiotherapy (CCRT) is recommended as the standard treatment in Western countries [1-5]. However, an approach using neoadjuvant chemotherapy (NAC) is widely applied clinically in Japan, South Korea, China, and Italy [6]. Irinotecan (CPT-11), a drug developed in Japan, is reportedly useful as monotherapy or in combination with cisplatin (CDDP) for recurrent cervical cancer [7, 8]. We previously reported the efficacy and safety of a treatment regimen involving a 28-day cycle of CDDP administered on day 1 and CPT- 11 on days 1,8 , and 15 [9].

From the aspect of reducing the time to surgical therapy as the primary treatment, we conducted a phase II clinical 
study of NAC in combination with radical hysterectomy with a dosing schedule employing a 21-day cycle with a higher than usual CDDP dose intensity.

\section{Subjects and methods}

\section{Subjects}

The subjects were 42 patients with stages IB2 to IIIB squamous cell carcinoma of the uterine cervix with a bulky mass. These patients gave informed consent and were scheduled to undergo radical hysterectomy during the period from June 2002 to March 2014.

\section{Justification for the target sample size}

We previously reported that the response rate to the CPT11/CDDP regimen [CPT-11 $60 \mathrm{mg} / \mathrm{m}^{2}$ (days $1,8,15$ ), CDDP $70 \mathrm{mg} / \mathrm{m}^{2}$ (day 1) q28 days] was $59 \%$ [9]. Accordingly, the threshold response rate was set at $50 \%$, because it would not be worthwhile to use this strategy in a clinical setting if the response rate was significantly lower than $50 \%$. The response rates to platinum-based NAC in patients with cervical cancer reportedly range from $76 \%$ to $95 \%[6,10,11]$. Accordingly, the expected response rate was set at $80 \%$, anticipating that the response rate to this regimen would be $80 \%$. The number of patients required was calculated to be 36 based on a presumed binominal distribution with a threshold value of $59 \%$, expected response rate of $80 \%$, and two-sided $\alpha$-level of 0.05 and $\beta$-level of $0.2(1-\beta=0.8)$. The number of planned subjects was thus set at 40 patients, taking into account the possibility of a few patients becoming ineligible or dropping out.

\section{Inclusion criteria}

The following set of inclusion criteria was employed for selection of study subjects. (1) Histologically verified squamous cell carcinoma of the uterine cervix; (2) age more than 20 years and less than 70 years; (3) locally advanced stage IB2 to IIIB; (4) Eastern Cooperative Oncology Group (ECOG) performance status (PS) 0-2; (5) initially treated case; (6) presence of a magnetic resonance imaging (MRI)-measurable bulky mass in the uterine cervix; (7) hematological and blood biochemical findings meeting the following criteria (WBC count $\geq 4,000 / \mathrm{mm}^{3}$; neutrophil count $\geq 2,000 / \mathrm{mm}^{3}$; platelet count $\geq 100,000 / \mathrm{mm}^{3}$; hemoglobin $\geq 10.0 \mathrm{~g} / \mathrm{dl}$; AST and ALT levels $\leq 2$ times the upper limit of normal reference range at study site; serum total bilirubin level $\leq 1.5 \mathrm{mg} / \mathrm{dl}$; serum creatinine $\leq 1.5 \mathrm{mg} / \mathrm{dl}$; and creatinine clearance $\geq 60 \mathrm{ml} / \mathrm{min}$ ); (8) life expectancy $\geq 6$ months; and (9) written informed consent personally given by the subject.

\section{Exclusion criteria}

Exclusion criteria were prescribed as follows. (1) Patients with overt infection; (2) patients with a serious complication (e.g., cardiac disease, poorly controlled diabetes mellitus, malignant hypertension, bleeding tendency); (3) patients with active multiple cancer; (4) patients with interstitial pneumonia or pulmonary fibrosis; (5) patients with effusions; (6) patients with a history of unstable angina or myocardial infarction within 6 months after registration, or with a concurrent serious arrhythmia requiring treatment; (7) patients for whom treatment with cisplatin and irinotecan is contraindicated; (8) patients with (watery) diarrhea; (9) patients with intestinal paralysis or ileus; (10) pregnant women, nursing mothers, or women wishing to become pregnant; (11) patients with a history of serious drug hypersensitivity or drug allergy; and (12) patients who were inadequate for safe conduct of this study as judged by the attending physician.

\section{Neoadjuvant chemotherapy}

CDDP at $70 \mathrm{mg} / \mathrm{m}^{2}$ was intravenously administered on day 1 and CPT- 11 at $70 \mathrm{mg} / \mathrm{m}^{2}$ was intravenously administered on days 1 and 8 of a 21-day cycle. In principle, two cycles were administered followed by radical hysterectomy.

\section{Dose modification criteria}

1. Criteria for CPT-11 dose skip. The CPT-11 dose on day 8 will be skipped if hematological test values within 2 days before day 8 fail to fulfill the following criteria: (1) neutrophil count $\geq 1,000 / \mathrm{mm}^{3}$, and (2) platelet count $\geq 75,000 / \mathrm{mm}^{3}$.

2. Criteria for initiation of the second cycle. Initiation of the second cycle will be postponed up to a maximum of 2 weeks if hematological test values within 2 days before the scheduled second cycle initiation day fail to fulfill the following criteria: (1) neutrophil count $\geq 1,500 / \mathrm{mm}^{3}$, (2) platelet count $\geq 75,000 / \mathrm{mm}^{3}$, and (3) serum creatinine $\leq 1.5 \mathrm{mg} / \mathrm{dl}$.

\section{Dose reduction criteria}

The doses of cisplatin and irinotecan will be reduced to 70 and $60 \mathrm{mg} / \mathrm{m}^{2}$, respectively, in the second course for patients for whom any of the following signs of toxicity is noted in the first cycle: (1) grade 4 neutropenia persisting for $\geq 7$ days, (2) febrile neutropenia persisting for $\geq 4$ days, 
(3) grade 4 thrombocytopenia, (4) grade 3 thrombocytopenia with hemorrhage, and (5) grade $\geq 3$ nonhematological toxicity excluding nausea, vomiting, appetite loss, fatigue, and hair loss.

\section{Supportive therapy}

Therapeutic administration of granulocyte-colony-stimulating factor (G-CSF) preparations was undertaken when grade 4 neutropenia was noted in the first cycle. In the second cycle and thereafter, prophylactic use of the preparation in patients with grade 3 neutropenia was acceptable if grade 4 neutropenia had been noted in the first cycle. Antiemetics were used for preventive purposes.

\section{Endpoints/variables}

The primary endpoint was antitumor efficacy, and the secondary endpoints were adverse events, completion rate of radical hysterectomy, operative time, surgical blood loss, progression-free survival (PFS), and overall survival (OS). Antitumor efficacy, PFS, and OS were also calculated by stage.

For the determination of antitumor efficacy, MRI was performed after the completion of course 1 and course 2, using MRI images before treatment as the baseline. Antitumor efficacy was determined using the Response Evaluation Criteria in Solid Tumors (RECIST) version 1.1, with response being the best evaluation. Adverse events were evaluated according to the National Cancer Institute Common Toxicity Criteria (NCI-CTCAE) version 4.0.

\section{Main treatment}

Stage Ib2-IIIb patients were subjected to a radical hysterectomy unless the antitumor response was progression of disease or up-stage progression. A radical hysterectomy was performed on stage IIIb patients with a down-stage progression. As a rule, lymph node dissection included the lymph nodes within the pelvis (external iliac nodes, internal iliac nodes, common iliac nodes, suprainguinal nodes, parametrial nodes, and obturator nodes). Concurrent chemoradiation therapy (CCRT) was carried out for patients whose conditions were inoperable.

\section{Postoperative adjuvant therapy}

Patients with a positive surgical margin, metastatic lymph nodes, infiltration to the parametrium, and/or vascular invasion, as demonstrated by pathological examination of the resected specimens, underwent postoperative irradiation, chemotherapy or CCRT. Before 2008, irradiation was performed as postoperative adjuvant therapy, whereas chemotherapy was performed from 2008 onward. However, CCRT was performed for patients who had multiple lymph node metastases and/or infiltration to the parametrium.

\section{Statistical analysis}

Progression-free survival and overall survival were calculated from the date of start of NAC, to the documented date of progression, death, or last follow-up, whichever occurred first. Impact of surgery result on survival was assessed by constructing Kaplan-Meier curves with a logrank test. Cox regression analyses were performed to assess the prognostic factors on survival. All reported significance was two tailed at a level of 0.05 .

\section{Results}

\section{Clinical characteristics}

Among 43 patients enrolled, 1 was diagnosed as having glassy cell carcinoma based on the postoperative histopathological examination. Therefore, we analyzed the other 42 patients. The median age was 45 years (range, 25-63). The performance status was 0 in 37 patients and 1 in 5, and clinical progression was stage IB2 in 9 patients, stage IIA2 in 2, stage IIB2 in 27, and stage IIIB in 4. Histological subtypes were keratinizing type in 11 patients and nonkeratinizing type in 31. Computed tomography before NAC showed lymph node metastasis in 16 patients and no lymph node metastasis in 26. The tumor size was less than $5 \mathrm{~cm}$ in 18 patients and $5 \mathrm{~cm}$ or more in 24 by MRI before NAC. Thirteen patients were positive and 29 patients were negative for pathological lymph node metastasis. Postoperative treatments were radiation therapy in 13 patients, chemotherapy in 15 , and chemoradiotherapy in 3 ; no postoperative treatment was given to 9 patients (Table 1 ).

\section{Response}

The antitumor effect was complete response (CR) in 7 patients, partial response (PR) in 28, stable disease (SD) in 6 , and progressive disease (PD) in 1, and the response rate was $83.3 \%$ [95 \% confidence interval (CI), 68.6-93.0]. Response rates by progression were $100 \%$ for stage I, $82.8 \%$ for stage II, and $50.0 \%$ for stage III (Table 2 ).

\section{Adverse events}

Grades 3 and 4 neutropenia were observed in $11(26.2 \%)$ and $12(28.6 \%)$ patients, respectively. Grade 3 or more severe anemia was noted in 4 patients $(9.5 \%)$; among them, a patient with grade 4 anemia required a blood transfusion. 
Table 1 Patient characteristics

\begin{tabular}{ll}
\hline Characteristic & $n=42$ \\
\hline Age (years) & \\
$\quad$ Median (range) & \\
PS & $37 / 5$ \\
0/1 & \\
FIGO stage & $9 / 29 / 4$ \\
1/11/III & \\
Histological type & $11 / 31$ \\
Keratinizing type/non-keratinizing type & \\
Preoperative lymph node metastasis & $16 / 26$ \\
Positive/negative & \\
Tumor diameter & $24 / 18$ \\
$>5$ cm/<5 cm & \\
Pathological lymph node metastasis & $13 / 29$ \\
Positive/negative & \\
Postoperative treatment & 9 \\
None & 13 \\
Radiation & 15 \\
Chemotherapy & 3 \\
Chemoradiotherapy &
\end{tabular}

Table 2 Response $(n=42)$

\begin{tabular}{lcrllc}
\hline & CR & PR & SD & PD & Objective response $(\mathrm{CR}+\mathrm{PR})$ \\
\hline Total $(n=42)$ & 7 & 28 & 6 & 1 & $35(83.3 \%)$ \\
Stage 1 $(n=9)$ & 2 & 7 & 0 & 0 & $9(100 \%)$ \\
Stage 11 $(n=29)$ & 2 & 20 & 4 & 1 & $24(82.8 \%)$ \\
Stage 111 $(n=4)$ & 1 & 1 & 2 & 0 & $2(50.0 \%)$ \\
\hline
\end{tabular}

$C R$ complete response, $P R$ partial response, $S D$ stable disease, $P D$ progressive disease

Grade 3 or more severe decreases in platelet counts were observed in 1 patient $(2.4 \%)$. As grade 3 or more severe nonhematological toxicity, grade 3 nausea occurred in 3 patients $(7.1 \%)$, vomiting in $1(2.4 \%)$, and grade 3 febrile neutropenia in $2(7.1 \%)$ (Table 3$)$.

Among the total 42 patients, $40(95.2 \%)$ completed chemotherapy as scheduled. The administration of CPT-11 on day 8 of cycle 2 was skipped in the remaining 2 patients. Grade 3 nausea persisted in these 2 patients; although it did not satisfy the skip criteria, in both cases the primary physician decided to skip the administration. The administration in cycle 2 was postponed in 3 patients $(10.7 \%)$ because neutrophil counts did not satisfy the therapy initiation criteria; however, all these 3 patients were able to receive treatment within 7 days. In 2 patients $(10.0 \%)$ in whom febrile neutropenia continued for 4 days or more, the doses of CPT-11 and CDDP were reduced from 70 to $60 \mathrm{mg} / \mathrm{m}^{2}$.
Table 3 Adverse events $(n=42)$

\begin{tabular}{|c|c|c|c|c|c|}
\hline & Grade 1 & Grade 2 & Grade 3 & Grade 4 & Grade $>3$ \\
\hline \multicolumn{6}{|c|}{ Hematological toxicity } \\
\hline Leukopenia & 8 & 20 & 8 & 3 & $11(26.2)$ \\
\hline Neutropenia & 4 & 14 & 11 & 12 & $23(54.8)$ \\
\hline Anemia & 17 & 20 & 3 & 1 & $4(9.5)$ \\
\hline $\begin{array}{l}\text { Thrombocyto- } \\
\text { penia }\end{array}$ & 10 & 2 & 1 & 0 & $1(2.4)$ \\
\hline \multicolumn{6}{|c|}{ Nonhematological toxicity } \\
\hline Nausea & 25 & 9 & 3 & 0 & $3(7.1)$ \\
\hline Vomiting & 21 & 7 & 1 & 0 & $1(2.4)$ \\
\hline Diarrhea & 4 & 2 & 0 & 0 & $0(0)$ \\
\hline Creatinine & 2 & 0 & 0 & 0 & $0(0)$ \\
\hline $\begin{array}{l}\text { Febrile neutro- } \\
\text { penia }\end{array}$ & 0 & 0 & 2 & 0 & $2(4.8)$ \\
\hline
\end{tabular}

\section{Operative details}

Among the total 42 patients, 2 with stage IIIB had inoperable disease. Forty patients underwent surgery. Radical hysterectomy was performed for 38 patients, but in 1 of them surgery was incomplete because of difficulty in resecting the lymph node metastasis. Simple total hysterectomy and exploratory laparotomy were performed in 1 patient each. The operable rate of surgery was $95.2 \%$; completion rate of radical hysterectomy was $88.1 \%$ (Fig. 1). Median number of lymph nodes dissected was 22 (range, 3-49); median operative time was $260 \mathrm{~min}$ (range, 210-334); median blood loss was $500 \mathrm{ml}$ (range, 393-898). Blood transfusions were given to 6 of 40 patients $(15.0 \%)$. The median time from surgery to discharge was 21 days (range, 16-26) (Table 4).

\section{PFS and OS analysis}

Of the 38 patients who underwent radical hysterectomy (excluding 1 patient who underwent probelaparotomy), 10 patients $(26.3 \%)$ developed tumor recurrence, that is, recurrence was noted in $1(50.0 \%)$ of the 2 patients who received postoperative CCRT, $2(13.3 \%)$ of the 15 patients who received postoperative chemotherapy, $5(41.7 \%)$ of the 12 patients who received postoperative radiotherapy, and $2(22.2 \%)$ of the 9 patients who did not receive postoperative adjuvant therapy (Table 5). Tumor recurrence in the lymph nodes was noted in 4 patients (affecting the paraaortic lymph nodes in all 4 cases and accompanied by metastasis to the subclavicular lymph nodes in 1 case). Of the 4 patients unable to undergo radical hysterectomy, 1 patient underwent CCRT after a simple total hysterectomy. CCRT was used for the patient who underwent 
Fig. 1 Operation consort diagram. SCC squamous cell carcinoma, NAC neoadjuvant chemotherapy

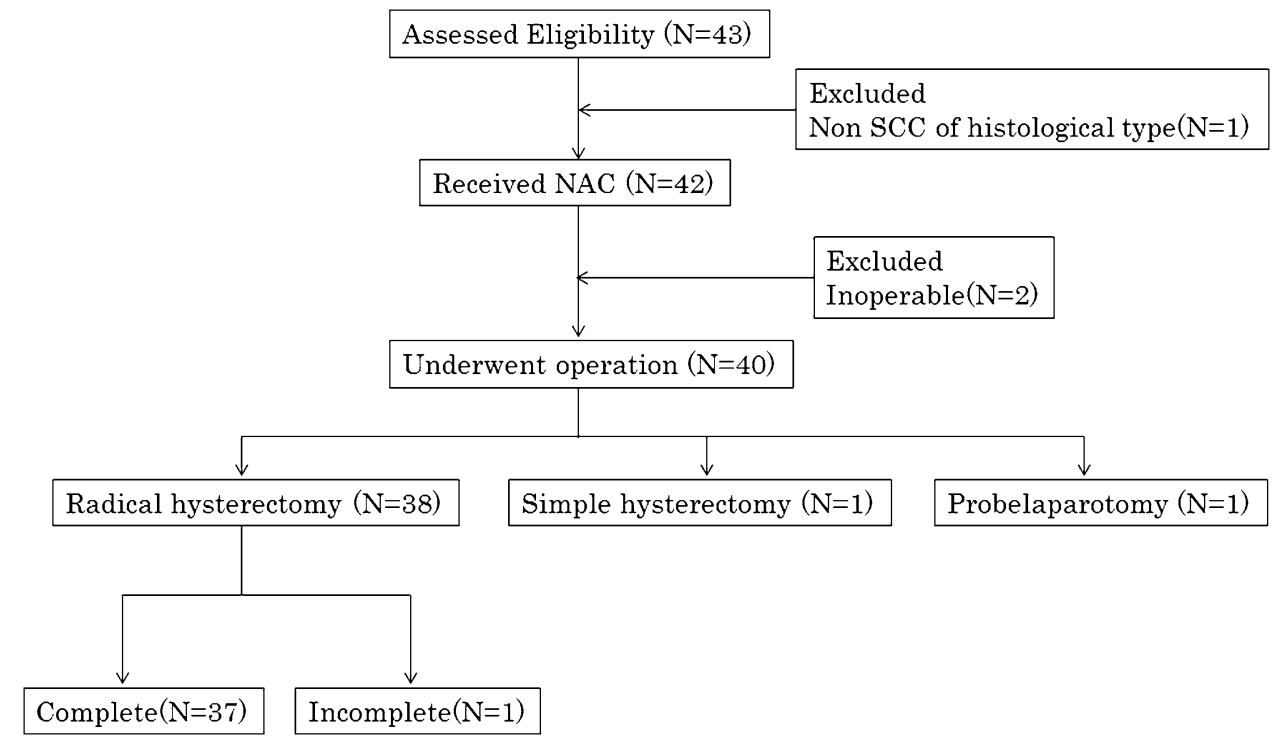

Table 4 Operative details

\begin{tabular}{ll}
\hline Characteristic & $n=42$ \\
\hline Type of surgery & 40 \\
RH & 38 \\
TAH & 1 \\
Probe laparotomy & 1 \\
Inoperable & 2 \\
Operation time (min) & 260 (210-334) \\
Median (IQR) & \\
Blood loss (ml) & 500 (393-898) \\
Median (IQR) & \\
Blood transfusion & 6 \\
Yes & 34 \\
No & \\
Time from surgery to discharge (Days) & 21 (16-26) \\
Median (IQR) & \\
Pathological lymph node metastasis & 13 \\
Positive & 29 \\
Negative &
\end{tabular}

$R H$ radical hysterectomy, TAH total abdominal hysterectomy, IQR interquartile range

probelaparotomy, and CCRT and radiation were used for the 2 patients judged unable to receive post-NAC surgery, but all 3 of these patients developed intrapelvic recurrence.

The median follow-up period for 42 patients was 45 months (range, 8-143), and the 5-year PFS rate and 5 -year OS rate were $67.2 \%$ and $68.0 \%$, respectively. As to progression, the 5-year PFS rates for patients at stages I, II, and III were $53.3 \%, 78.4 \%$, and $25.0 \%$, respectively, and the 5-year OS rates were $53.3 \%, 79.2 \%$, and $25.0 \%$, respectively (Figs. 2, 3). In multivariate analysis of OS, lymph node metastasis before NAC [hazard radio (HR), $34.88 ; p=0.0031$ ] and response of NAC (HR, 30.58; $p=0.0014)$ were extracted as significant prognosis factors (Table 6).

\section{Discussion}

Clinical studies of NAC for various forms of cancer are underway, but as yet there is little evidence of the usefulness of NAC in the field of gynecology including cervical, endometrial, and ovarian cancers. The clinical significance of NAC for cervical cancer is that the following can be expected: (1) tumor size reduction, which improves operative curability and safety and thus expands the surgical indications; and (2) inhibition of distant metastasis based on systemic effects being exerted on latent and lymph node micrometastatic lesions. As such, NAC for cervical cancer in Japan is currently performed to improve operative curability and safety as well as to increase the indications for surgery. However, there are no reports indicating that NAC prolongs survival.

A key drug for progressive recurrent cervical cancer is CDDP; the results of a randomized study by the Gynecologic Oncology Group (GOG) demonstrated the usefulness of paclitaxel and topotecan in addition to CDDP, and dual therapy with CDDP has been recommended for recurrence and progressive cancer $[12,13]$. Topotecan is widely used in Western countries, whereas mainly CPT-11, with a mechanism of action based on the same DNA type I topoisomerase inhibitory action, is used in Japan.

In a study of combination therapy with CDDP [CDDP $60 \mathrm{mg} / \mathrm{m}^{2}$ (day 1$)+$ CPT-11 $60 \mathrm{mg} / \mathrm{m}^{2}$ (days $\left.\left.1,8,15\right)\right]$, the response rate in patients with recurrent/progressive cervical 
Table 5 Details of recurrent patients after radical hysterectomy $(n=38)$

\begin{tabular}{llll}
\hline Adjuvant therapy & Number & Rate $(\%)$ & Recurrent sites \\
\hline Chemoradiotherapy $(n=2)$ & 1 & 50.0 & PAN (1) \\
Chemotherapy $(n=15)$ & 2 & 13.3 & PAN (1), pelvic cavity (1) \\
Radiation $(n=12)$ & 5 & 41.7 & PAN (2), subclavicular lymph node (1), pelvic cavity (3) \\
No therapy $(n=9)$ & 2 & 22.2 & Vaginal stump (2) \\
\hline
\end{tabular}

PAN paraaortic lymph node cancer was $59.9 \%$, and the response rate when this regimen was administered as NAC was $78 \%[6,9]$. To reduce the time to surgical therapy, the primary treatment, we conducted a phase II clinical study of NAC in combination with radical hysterectomy with a dosing schedule involving a 21-day cycle with a higher than usual CDDP dose intensity. The response rate in 42 patients was $83.3 \%$, better than the therapeutic results of Sugiyama et al. Notably, a

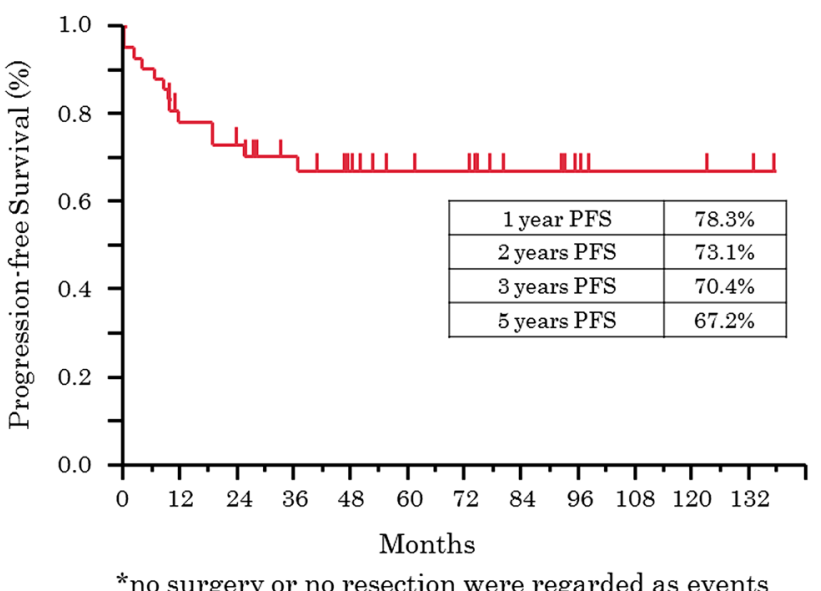

Fig. 2 Kaplan-Meier plot of progression-free survival $(n=42)$

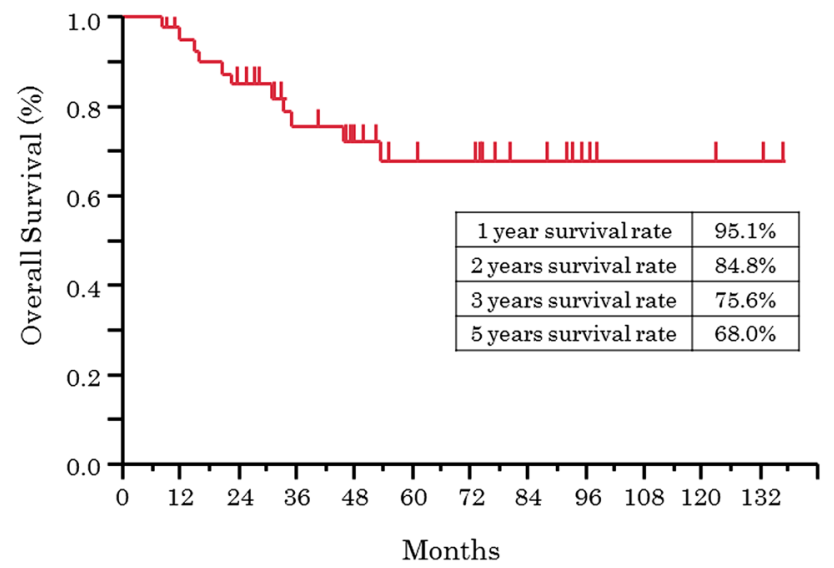

Fig. 3 Kaplan-Meier plot of overall survival $(n=42)$ response rate of $86.8 \%$ was obtained in 38 patients with stage IB2 to IIB disease. However, the response rates were $50.0 \%$ in 4 stage IIIB patients, $50 \%$ in operated patients, and $25 \%$ in patients who had completed radical hysterectomy. Our results indicate that NAC plus radical hysterectomy is not useful in stage III patients.

With regard to adverse events, the incidence of grade 3 or more severe neutropenia was $54.8 \%$, although this adverse event could be managed with a G-CSF agent. In all cases with diarrhea characteristic of CPT-11, the severity was grade 2 or lower. A single dose of CPT-11 had to be reduced in this regimen because of administration in divided doses on days 1 and 8 , thereby preventing grade 3 or more severe diarrhea.

In 40 patients undergoing NAC plus radical hysterectomy, median operative time was 260 min and median surgical blood loss was $500 \mathrm{ml}$, similar to those findings in studies of radical hysterectomy for patients with stage IB2 to IIB with a bulky mass reported by $\mathrm{He}$ et al. and Wang et al. $[14,15]$. No serious postoperative complications, such as intestinal obstruction and thrombosis, occurred. Accordingly, this study demonstrated the safety of NAC plus radical hysterectomy, with no major adverse effects for patients.

We compared the treatment outcomes in our 38 IB2-IIB cases with the results reported by Uegaki et al. [16]. The response rate for our cases was $86.6 \%$, comparable to the rate reported by Uegaki et al. (86.2\%). The 5-year PFS (73.3\% vs. $62.2 \%)$ and 5-year OS (78.9\% vs. $74.9 \%)$ were better in our cases than in the cases reported by Uegaki et al. Comparisons were also made with respect to adjuvant therapy and recurrence sites. According to the report by Uegaki et al., recurrence was noted in 21 (32.3\%) of the 65 cases, and 15 of these 21 cases had received adjuvant therapy, but recurrence in the pelvic cavity was observed in only 4 cases (the other cases of recurrence had remote metastasis, including metastasis to the paraaortic lymph nodes). Of the 38 patients we managed, 10 patients $(26.3 \%)$ developed recurrence, including 8 patients who received adjuvant therapy (4 patients with intrapelvic recurrence and the other 4 patients with metastasis to lymph nodes, including the paraaortic lymph nodes). Postoperative adjuvant therapy by means of CCRT or radiation was performed in $27(93.1 \%)$ of the 29 cases reported by Uegaki et al. and $14(50.0 \%)$ of the 28 cases managed by us. Postoperative 
Table 6 Multivariate analysis of treatment-related factors for overall survival (OS)

\begin{tabular}{lllll}
\hline Factor & & Hazard ratio & $95 \%$ CI & $p$ value \\
\hline PS & $0 / 1$ & 0.11 & $0.002-2.693$ & 0.1858 \\
FIGO stage & Stage I, II/III & 5.75 & $0.224-90.468$ & 0.2435 \\
Tumor diameter & $>5 /<5$ & 0.25 & $0.009-3.177$ & 0.2788 \\
Pre-NAC lymph node metastasis & Positive/negative & 34.88 & $2.969-1152.9$ & 0.0031 \\
Tumor response & CR, PR/SD, PD & 30.59 & $3.675-447.9$ & 0.0014 \\
Pathological lymph node metastasis & Positive/negative & 1.21 & $0.182-7.932$ & 0.836 \\
Postoperative treatment & Yes/no & 6.81 & $0.718-174.323$ & 0.0983 \\
\hline
\end{tabular}

$N A C$ neoadjuvant chemotherapy
CCRT or radiation allowed control of intrapelvic recurrence to some extent, whereas postoperative chemotherapy tended to reduce the risk of remote recurrence. These results suggest that the site of recurrence depends on the type of postoperative adjuvant therapy. Furthermore, as recurrence was also observed in cases that did not receive postoperative adjuvant therapy, it seems advisable to perform postoperative adjuvant therapy in all cases of bulky tumors.

Six randomized clinical trials (1036 patients) including Study JCOG102 in Japan were analyzed in Cochrane Reviews in 2010, and NAC in combination with surgical therapy reportedly improved the disease-free survival rate as compared with initial surgery (HR, 0.76; $p=0.01$ ). NAC plus radical hysterectomy was thus suggested to improve patient prognosis [17]. According to a multivariate analysis of OS, clinical stages, lymph node metastasis before NAC, and antitumor effects are significant prognostic factors. Therefore, it was suggested that NAC plus radical hysterectomy, for patients other than those with lymph node metastasis before NAC, may further improve the therapeutic effects of NAC. That is, the prognosis of patients with stage IB2 to IIB cervical cancer with a bulky mass might be improved by hysterectomy alone in those who have lymph node metastasis before NAC, and by NAC plus radical hysterectomy in those without lymph node metastasis. The next step we should take would be implementation of a phase II clinical study on the use of NAC plus radical hysterectomy plus adjuvant therapy, with details of postoperative adjuvant therapy set forth in advance, in patients having a bulky tumor (clinical stage IB2-IIB). The results from such a study would provide important data for planning a phase III clinical study on a national scale of NAC plus radical hysterectomy plus adjuvant therapy versus radical hysterectomy plus adjuvant therapy versus CCRT.

\section{Conclusions}

The results of this study suggest that NAC, that is, the CDDP/CPT-11 regimen, in combination with radical hysterectomy exerts high antitumor efficacy with manageable adverse reactions. Thus, NAC may facilitate safe radical hysterectomy. This treatment strategy is considered to be therapeutically useful, and an improved prognosis can also be expected. Therefore, we add our evidence of the usefulness of NAC plus radical hysterectomy in Japan to the world literature on cervical cancer treatment.

\section{Compliance with ethical standards}

Conflict of interest None of the authors of this manuscript has any conflicts of interest to declare.

Open Access This article is distributed under the terms of the Creative Commons Attribution 4.0 International License (http://creativecommons.org/licenses/by/4.0/), which permits unrestricted use, distribution, and reproduction in any medium, provided you give appropriate credit to the original author(s) and the source, provide a link to the Creative Commons license, and indicate if changes were made.

\section{References}

1. Pearcey R, Brundage M, Drouin P et al (2002) Phase III trial comparing radical radiotherapy with and without cisplatin chemotherapy in patients with advanced squamous cell cancer of the cervix. J Clin Oncol 20:966-972

2. Eifel PJ, Winter K, Morris M et al (2004) Pelvic irradiation with concurrent chemotherapy versus pelvic and para-aortic irradiation for high-risk cervical cancer: an update of Radiation Therapy Oncology Group trial (RTOG) 90-01. J Clin Oncol 22:872-880

3. Green JA, Kirwan JM, Tierney JF et al (2001) Survival and recurrence after concomitant chemotherapy and radiotherapy for cancer of the uterine cervix: a systematic review and meta-analysis. Lancet 358:781-786

4. Lukka H, Hirte H, Fyles A et al (2002) Concurrent cisplatinbased chemotherapy plus radiotherapy for cervical cancer: a meta analysis. J Clin Oncol 14:203-212

5. NCCN (2000) Clinical practice guidelines in oncology: cervical cancer, vol 2. National Comprehensive Cancer Network,

6. Sugiyama T, Nishida T, Kumagai $S$ et al (1999) Combination chemotherapy with irinotecan and cisplatin as neoadjuvant in locally advanced cervical cancer. Br J Cancer 81:95-98

7. Sugiyama T, Nishida T, Kataoka A et al (1996) Combination of irinotecan hydrochloride (CPT-11) and cisplatin as a new 
regimen for patients with advanced ovarian cancer. Acta Obstet Gynecol Jpn 48:827-834

8. Takeuchi S, Dobashi K, Fujimoto S et al (1991) A late phase II study of CPT-11 on uterine cervical cancer and ovarian cancer. Research Groups of CPT-11 in Gynecologic Cancers. Gan To Kagaku Ryoho 18:31-37

9. Sugiyama T, Yakushiji M, Noda K et al (2000) Phase II study of irinotecan and cisplatin as first-line chemotherapy in advanced or recurrent cervical cancer. Oncology 58:31-37

10. Dueñas-Gonzalez A, López-Graniel C, González- A et al (2001) A phase II study of gemcitabine and cisplatin combination as induction chemotherapy for untreated locally advanced cervical carcinoma. Ann Oncol 12:541-547

11. D'Agostino G, Distefano M, Greggi S et al (2002) Neoadjuvant treatment of locally advanced carcinoma of the uterine cervix with epirubicin, paclitaxel and cisplatin. Cancer Chemother Pharmacol 49:256-260

12. Rose PG, Blessing JA, Gershenson DM et al (1999) Paclitaxel and cisplatin as first-line therapy in recurrent or advanced squamous cell carcinoma of the cervix: a Gynecologic Oncology Group study. J Clin Oncol 17:2676-2680
13. Moore DH, Blessing JA, McQuellon RP et al (2004) Phase III study of cisplatin with or without paclitaxel in stage IVB, recurrent, or persistent squamous cell carcinoma of the cervix. a Gynecologic Oncology Group study. J Clin Oncol 22:3113-3119

14. He Du, Duan Chunyan, Chen Jun et al (2015) The safety and efficacy of the preoperative neoadjuvant chemotherapy for patients with cervical cancer: a systematic review and meta analysis. Int J Clin Exp Med 8:14693-14700

15. Wang Y, Wang G, Wei LH et al (2011) Neoadjuvant chemotherapy for locally advanced cervical cancer reduces surgical risks and lymph-vascular space involvement. Chin J Cancer 30:645-654

16. Uegaki K, Shimada M, Sato S et al (2014) Outcome of stage IB2-IIB patients with bulky uterine cervical cancer who underwent neoadjuvant chemotherapy followed by radical hysterectomy. Int J Clin Oncol 19:348-353

17. Chemoradiotherapy for Cervical Cancer Meta-analysis Collaboration (CCCMAC) (2010) Reducing uncertainties about the effects of chemoradiotherapy for cervical cancer: individual patient data meta-analysis. Cochrane Database Syst Rev 20:CD008285 